\title{
EDITORIALS
}

\section{Diagnostic Performance of Multislice CCTA: Is it Ready for Clinical Use}

\author{
Rita F Redberg, MD, MSC \\ School of Medicine, Division of Cardiology, University of California, San Francisco, San Francisco, CA, USA.
}

$\mathrm{J}$ Gen Intern Med 26(3):230-1

DOI: $10.1007 / \mathrm{s} 11606-010-1627-\mathrm{Z}$

(C) The Author(s) 2011. This article is published with open access at Springerlink.com

\begin{abstract}
A dvances in CT technology have expanded our ability to noninvasively image the coronary arteries. The use of CT scanning has increased significantly in the last few years. Efforts to synthesize and interpret clinical trial data to best inform the rational clinical use of coronary computed tomographic angiography (CCTA) need to keep pace with the increased utilization of this promising technology.

In this issue of JGIM, Ollendorf et al. present a scholarly and thoughtful systematic review of the literature of CCTA's diagnostic accuracy and the impact of CCTA on clinical decision-making and outcomes. ${ }^{1}$ They find that the pooled mean sensitivity is $98 \%$ and specificity is $85 \%$ for the 42 diagnostic studies of CCTA current to February 2010. However, these findings must be viewed in light of significant limitations. These limitations lie not in the quality of this excellent analysis, but in the meager and narrow data that supports our current use of cardiac CTA for diagnosis of patients with suspected $\mathrm{CAD}$. The authors find that "spectrum bias" (which the authors define as "the systematic variation of diagnostic test performance across patient subgroups coupled with the failure to present all such subgroups in a study") was present in the majority of CCTA studies. The existing data for cardiac CT derives almost entirely from patients already scheduled for invasive coronary angiography, where pretest probability is quite high. Indeed, even though most of the studies excluded patients with known CAD or a history of revascularization, the mean prevalence of CAD in all CCTA studies was an astonishingly high 59.3\%. By comparison, in a recent report involving the National Cardiovascular Data Registry, only $37.6 \%$ of patients referred for elective coronary angiography exhibited obstructive CAD. ${ }^{2}$ Clearly, the population of patients studied in the CCTA trials does not resemble the average American referred for coronary angiography. Furthermore, there is an even more striking disconnect between the populations included in these diagnostic accuracy trials of CCTA and the low to intermediate risk pretest probability of $\mathrm{CAD}$ patients-patients that published clinical guidelines consider the appropriate population for the use of CCTA for CAD detection. ${ }^{3}$ As Bayes' theorem tells us, the accuracy of diagnostic tests decreases significantly in a population with a lower pretest probability of disease. ${ }^{4}$ This difference in the populations where CCTA is studied and the populations in which CCTA is considered appropriate for use means that it is highly likely that the diagnostic accuracy of CCTA in clinical
\end{abstract}

Published online January 29, 2011 use has been overestimated. Unfortunately, the low pretest probability population (the population in which cardiac CTA is commonly used in actual clinical practice) is precisely the population where clinical trial data is sorely lacking. Conclusions from systematic reviews, even high quality ones such as that of Ollendorf et al., necessarily are limited due to the restrictions in the data that comprises them.

Although it is common for tests or therapies to be studied first in high risk populations, it is essential that they also be evaluated in the much larger lower risk population in which they will be used most commonly prior to widespread use of the new technology. New tests, such as cardiac CT, which has not been studied in a low probability population, should be used only in the context of a clinical trial until appropriate data can be collected and analyzed. A recent case report of a 52-year-old woman with hypertension, who had sharp nonexertional chest pain reproducible on palpation, illustrates the importance of a clear understanding of risks and benefits for cardiac CT imaging in a low pretest probability population. This unfortunate woman's doctor ordered a cardiac CT, simply to "reassure" her-a decision that led to disastrous consequences. ${ }^{5}$ Our enthusiasm for tests, especially for mere "reassurance," should not displace the substantial reassurance provided simply by talking to our patients. The temptation to order an imaging test for patients with a low probability of CAD is due, in part, to a misconception of the usefulness of a diagnostic test where the pretest probability of disease is minimal. The fact that many diagnostic tests are not without their own risks urges even more wisdom in their use. Although a single case report cannot tell us the frequency of use of cardiac CT for such low probability patients, it nevertheless serves as a strong cautionary tale.

There are many factors contributing to the rapidly increased use of CT imaging related to an overestimation of the benefits of this technology and an under appreciation of the risks, as well as the rapid increase in US-based cardiology practices that own or lease cardiac CT equipment, ${ }^{1}$ as noted by Ollendorf. The systematic review by Ollendorf et al. highlights the limitations of the current data on diagnostic accuracy, revealing there are no substantial data from studies of patients with low and intermediate pretest probability of CAD (the most common use of cardiac CT currently). The danger of a seemingly harmless noninvasive test like a CT scan is that it is easy for patients and physicians to be unaware of the potentially serious negative consequences of this test. False positives increase as the prevalence of true disease decreases in the population studied for any diagnostic test. False positives for a cardiac CTA mean that additional testing, often invasive coronary angiography (ICA), will be performed, as in the above case report. In addition to false positives, cardiac CT scans can have real and potentially dangerous consequences from apparent 'incidentalomas.' A recent study found that $40 \%$ of research imaging exams on otherwise healthy people had incidental findings that led to 
clinical action, often of unclear benefit. ${ }^{6}$ In addition, we know that there are significant radiation risks associated with CT scans, particularly in young and middle-aged patients whose lifetime risk for cancer from cumulative CT scans is higher. ${ }^{7,8}$

Ollendorf et al. also summarize the limited data for cardiac CT for clinical decision-making and outcomes. Only two studies compared CCTA to standard care in a total of 255 patients with 6- to 12-month follow-up. There were no major cardiovascular events in either group, although the revascularization rate was much higher in the CCTA group. One cannot conclude much from these preliminary studies besides the fact that these were very low risk patients and that more studies with longer follow-up are needed. Although this population of low to intermediate risk emergency department patients is of greatest interest for CCTA, it currently has little data to support its use.

Wise use of cardiac CT imaging, like any test or therapy, involves a thoughtful consideration of risks and benefits, and hopefully a frank discussion of these with the patient. To have a truly informed discussion, we must have high quality data on these risks and benefits. Ollendorf et al. do an excellent job on showing us where we are currently, and how far we have to go.

Disclosures: I am a member of the Institute for Clinical and Economic Review Advisory Board and was a member of the 17-person advisory committee for the ICER review of CCTA.

Open Access: This article is distributed under the terms of the Creative Commons Attribution Noncommercial License which permits any noncommercial use, distribution, and reproduction in any medium, provided the original author(s) and source are credited.
Corresponding Author: Rita F Redberg, MD, MSC; School of Medicine, Division of Cardiology, University of California, San Francisco, San Francisco, CA, USA (e-mail: redberg@medicine.ucsf. edu).

\section{REFERENCES}

1. Ollendorf D, Kuba M, Pearson S. Diagnostic performance of multi-slice CCTA, JGIM 2010. doi:10.1007/s11606-010-1556-x (Epub ahead of print).

2. Patel M, Peterson E, Dai D et al. Low diagnostic yield of elective coronary angiography. NEJM. 2010;362.

3. Taylor AJ, Cequeira M, Hodgson JM, Mark D, Min J, O'Gara P, Rubin GD. ACCF/SCCT/ACR/AHA/ASE/ASNC/SCAI/SCMR 2010 appropriate use criteria for cardiac computed tomography: a report of the American Collegeof Cardiology Foundation Appropriate Use Criteria Task Force, the Society of Cardiovascular Computed Tomography, the American College of Radiology, the American Heart Association, the American Society of Echocardiography, the American Society of Nuclear Cardiology, the Society for Cardiovascular Angiography and Interventions, and the Society for Cardiovascular Magnetic Resonance. J Am Coll Cardiol 2010;56:1864-94

4. Diamond GA, Kaul S. Prior convictions: Bayesian approaches to the analysis and interpretation of clinical megatrials. J Am Coll Cardiol. 2004;43:1929-39.

5. Becker MC, Galla, Nissen S. Left main trunk coronary artery dissection as a consequence of inaccurate coronary computed tomographic angiography. Arch Intern Med. 2010 (Epub ahead of print)

6. Orme $\mathbf{N}$ et al. Arch Int Med. 2010

7. Berrington de González A, Mahesh M, Kim K-P, et al. Projected cancer risks from computed tomographic scans performed in the United States in 2007. Arch Int Med. 2009;169(22):2071-2077.

8. Smith-Bindman R, Lipson J, Marcus R, et al. Radiation dose associated with common computed tomography examinations and the associated lifetime attributable risk of cancer. Arch Int Med. 2009; 169(22):2078-2086. 